Vol. 6, Issue 1, January 2019

\title{
Synthesis and Antimicrobial Activity of Arylazopyrazole Pyrimidone Clubbed Heterocyclic Compounds
}

\author{
Nikulsinh Sarvaiya ${ }^{1}$, Dr. Sheetal Gulati ${ }^{2}$, Dr. H. Patel ${ }^{3}$ \\ Dept. of Chemistry, Rabindranath Tagore University, Bhopal (M.P.), India ${ }^{1,2}$ \\ Ex-Head and Professor, Dept. of Chemistry, S.P. University, VV Nagar, Gujarat, India ${ }^{3}$
}

\begin{abstract}
Ethyl-3-oxo-2-(2-(4-sulfamoylphenyl)hydrazono) butanoate (2) on condensation with 6-methyl-2-oxo-4subsituted phenyl-1,2,3,4-tetrahydro pyrimidine-5-carbohydrazide (3a-e) to gave 4-(2-(3-methyl-1-(6-methyl-2-oxo-4subsituted phenyl-1,2,3,4-tetrahydro pyrimidine -5-carbonyl)-5-oxo-1H-pyrazol-4(5H)-ylidene) hydrazine) benzene sulfonamide (4a-e), which on reaction with benzaldehyde gives 4-(2-(1-(4-([1,1'-biphenyl]-4-yl)-3-(hydroxyl (phenyl) aryl)-6-methyl-2-oxo-1,2,3,4-tetrahydropyrimidine-5-carbonyl)-3-methyl-5-oxo-1H-pyrazol-4(5H)-ylidene)hydrazinyl) benzenesulfonamide( $5 \mathrm{a}-\mathrm{e})$.The structures of all these compounds $(4 \mathrm{a}-\mathrm{e})$ were recognized by analytical and spectral studies. The synthesized compounds were evaluated for their antimicrobial activity against various bacteria and fungi.
\end{abstract}

Keywords: Sulphadrug, Pyrazole and Antimicrobial Activity

\section{INTRODUCTION}

Sulpha drugs are bacteriostatic and are also referred to as antibacterial. The sulphonamides are synthetic antimicrobial agents with a wide spectrum encompassing most Gram-positive and many Gram-negative organisms. ${ }^{1-3}$

The heterocyclic compound, pyrimidinones shows various antimicrobial, hypnotic, antiviral, sedative, antineoplastic, anticonvulsant, analgesic and anti-inflammatory. ${ }^{4-9}$ The nitrogen containing pyrazole and its derivatives is show application in medicinal chemistry like antibactiral,antifungal,analgesic, anti-inflammatory, antipyretic, antiparasitic and antimalarial. ${ }^{10-14}$ The arylazopyrazoles are generally prepared by combination of aryl-azo-ethyl actoacetate derivatives and hydrazine derivatives, which shows biological activities including antibacterial, antifungicidal, analgesic, anti-inflammatory properties. ${ }^{15-17}$ These heterocyclic systems find wide use in medicine, agriculture and industry. Merging of both of arylazopyrazole and pyrimidone moieties into one molecule may enhance the drug activity to some extent, or may class of drug. Thus the objective of the present work is to explore new derivatives of pyrimidone containing arylazopyrazole of sulfa drug. The present communication comprises such concepts. So the whole synthetic approach is shown in scheme-1. 


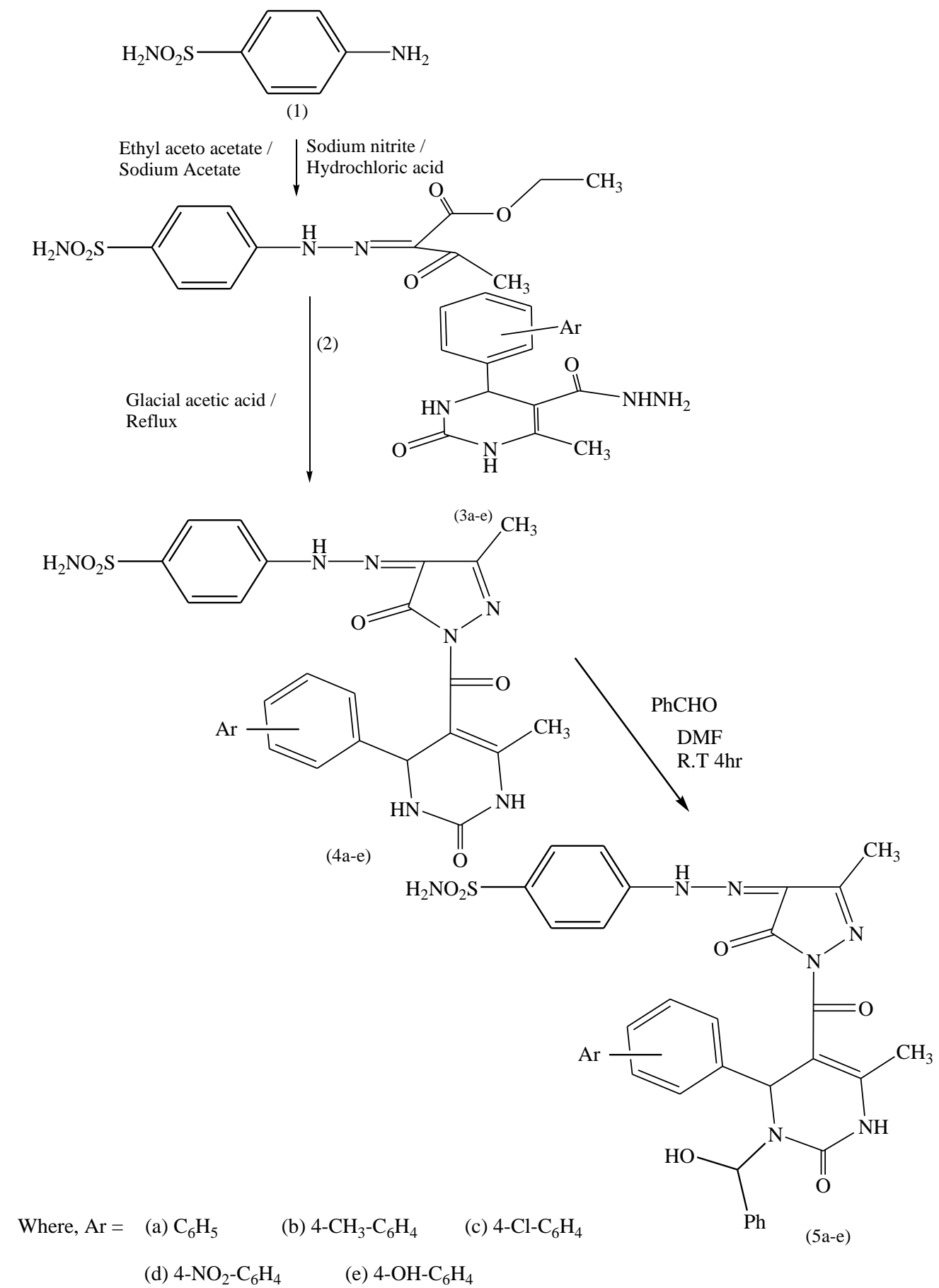

SCHEME-I

II. EXPERIMENTAL

The IR spectra were recorded in $\mathrm{KBr}$ pellets on a Nicolet 400D spectrometer. ${ }^{1} \mathrm{H}$ NMR spectra were recorded in DMSO with TMS as internal standard on a Bruker spectrometer at $400 \mathrm{MHz}$. LC-MS of selected samples taken on LC-MSDTrap-SL_01046. Purity of compound was checked by TLC on silica gel plates and the spots were visualized by UV lamp. 6-methyl-2-oxo-4-subsitutedphenyl-1,2,3,4-tetrahydro pyrimidine-5-carbo hydrazide (3a-e) were synthesis by reported method. ${ }^{18}$ The yields, melting points and other characterization data of these compounds are given in Table -1 . 


\section{International Advanced Research Journal in Science, Engineering and Technology}

Vol. 6, Issue 1, January 2019

A mixture of ethyl-3-oxo-2-(2-(4-sulfamoylphenyl)hydrazono)butanoate (2) and 6-methyl-2-oxo-4subsitutedphenyl-1,2,3,4-tetrahydro pyrimidine-5-carbohydrazide (3a-e) ${ }^{19,20}$ were mixed with glacial acetic acid and then refluxed for appropriate time. Then cooled and resulting solid was filtered off dried and crystallized from alcohol. The yields, melting points and other characterization data of these compounds are given in Table -2 .

Synthesis of 4-(2-(1-(4-([1,1'-biphenyl]-4-yl)-3-(hydroxy(phenyl)aryl)-6-methyl-2-oxo-1,2,3,4-tetrahydro pyrimidine-5-carbonyl)-3-methyl-5-oxo-1H-pyrazol-4(5H)-ylidene)hydrazinyl)benzenesulfonamide (5a-e)

In a round bottom flask a solution of 4-(2-(3-methyl-1-(6-methyl-2-oxo-4-subsitutedphenyl-1,2,3,4-tetrahydro pyrimidine -5-carbonyl)-5-oxo-1H-pyrazol-4(5H)-ylidene) hydrazinyl) benzene sulfonamide $(4 \mathrm{a}-\mathrm{e})(0.10 \mathrm{mmol})$ and benzaldehyde $(0.020 \mathrm{mmol})$ in $\mathrm{DMF}(50 \mathrm{ml})$ was taken and stirred by placing on magnetic stirrer at room temperature for 4 hours. The product was checked by TLC. The mixture was poured on crashed ice. The precipitates falled out. Filtered, Washed and air-dried. Repurified by ethanol. The yield was $65 \%$. The details are given in Table-3.

Table-1 Physical and Analytical Data of the Compounds Synthesized (3a-e)

\begin{tabular}{|c|c|c|c|c|c|c|c|c|c|c|}
\hline \multirow{3}{*}{$\begin{array}{l}\text { Comp. } \\
\text { No. }\end{array}$} & \multirow{3}{*}{$\begin{array}{l}\text { Molecular Formula } \\
\text { (Mol.wt.) }\end{array}$} & \multirow{3}{*}{$\begin{array}{c}\text { LC- } \\
\text { MS } \\
\text { Data }\end{array}$} & \multirow{3}{*}{$\underset{{ }^{\circ} \mathrm{C}}{\mathrm{M.P} *}$} & \multirow{3}{*}{$\underset{\%}{\text { Yield }}$} & \multicolumn{6}{|c|}{ Elemental Analysis } \\
\hline & & & & & \multicolumn{2}{|c|}{$\mathrm{C} \%$} & \multicolumn{2}{|c|}{$\mathrm{H} \%$} & \multicolumn{2}{|c|}{ N\% } \\
\hline & & & & & Calcd. & Found & Calcd. & Found & Calcd. & Found \\
\hline $\mathbf{3 a}$ & $\begin{array}{c}\mathrm{C}_{12} \mathrm{H}_{14} \mathrm{~N}_{4} \mathrm{O}_{2} \\
(246)\end{array}$ & 248 & $135-137$ & 78 & 58.53 & 58.5 & 5.73 & 5.7 & 22.75 & 22.7 \\
\hline $\mathbf{3 b}$ & $\begin{array}{c}\mathrm{C}_{13} \mathrm{H}_{16} \mathrm{~N}_{4} \mathrm{O}_{2} \\
(260)\end{array}$ & 263 & $142-144$ & 82 & 59.99 & 59.9 & 6.20 & 6.1 & 21.52 & 21.5 \\
\hline $3 c$ & $\begin{array}{c}\mathrm{C}_{12} \mathrm{H}_{13} \mathrm{~N}_{4} \mathrm{O}_{2} \mathrm{Cl} \\
(280)\end{array}$ & 295 & $130-132$ & 76 & 51.34 & 51.3 & 4.67 & 4.6 & 19.96 & 19.9 \\
\hline 3d & $\begin{array}{c}\mathrm{C}_{12} \mathrm{H}_{13} \mathrm{~N}_{5} \mathrm{O}_{4} \\
(291)\end{array}$ & 306 & $124-126$ & 80 & 49.48 & 49.48 & 4.50 & 4.4 & 24.04 & 24.0 \\
\hline $3 e$ & $\begin{array}{c}\mathrm{C}_{13} \mathrm{H}_{16} \mathrm{~N}_{4} \mathrm{O}_{3} \\
(276)\end{array}$ & 280 & $120-121$ & 69 & 56.51 & 56.4 & 5.84 & 5.8 & 20.28 & 20.2 \\
\hline
\end{tabular}

Table-2 Physical and Analytical Data of the Compounds Synthesized (4a-e)

\begin{tabular}{|c|c|c|c|c|c|c|c|c|c|c|c|c|}
\hline \multirow{3}{*}{$\begin{array}{l}\text { Comp. } \\
\text { No. }\end{array}$} & \multirow{3}{*}{$\begin{array}{l}\text { Molecular } \\
\text { Formula }\end{array}$} & \multirow{3}{*}{$\begin{array}{c}\text { LC- } \\
\text { MS } \\
\text { Data }\end{array}$} & \multirow{3}{*}{$\underset{{ }^{\circ} \mathrm{C}}{\text { M.P.* }}$} & \multirow{3}{*}{$\begin{array}{c}\text { Yield } \\
\%\end{array}$} & \multicolumn{8}{|c|}{ Elemental Analysis } \\
\hline & & & & & \multicolumn{2}{|c|}{$\mathrm{C} \%$} & \multicolumn{2}{|c|}{ H\% } & \multicolumn{2}{|c|}{$\mathrm{N \%}$} & \multicolumn{2}{|c|}{ S\% } \\
\hline & & & & & Calcd. & Found & Calcd. & Found & Calcd. & Found & Calcd. & Found \\
\hline $4 a$ & $\begin{array}{c}\mathrm{C}_{22} \mathrm{H}_{21} \mathrm{~N}_{7} \mathrm{O}_{5} \mathrm{~S} \\
(495)\end{array}$ & 502 & $\begin{array}{l}192- \\
194\end{array}$ & 62 & 53.33 & 53.3 & 4.27 & 4.2 & 19.79 & 19.7 & 6.47 & 6.4 \\
\hline $4 b$ & $\begin{array}{c}\mathrm{C}_{23} \mathrm{H}_{23} \mathrm{~N}_{7} \mathrm{O}_{5} \mathrm{~S} \\
(509)\end{array}$ & 512 & $\begin{array}{l}197- \\
199 \\
\end{array}$ & 65 & 54.22 & 54.2 & 4.55 & 4.5 & 19.24 & 19.2 & 6.29 & 6.2 \\
\hline $4 c$ & $\begin{array}{c}\mathrm{C}_{22} \mathrm{H}_{20} \mathrm{~N}_{7} \mathrm{O}_{5} \mathrm{SCl} \\
(529)\end{array}$ & 536 & $\begin{array}{l}198- \\
201\end{array}$ & 60 & 49.86 & 49.8 & 3.80 & 3.7 & 18.50 & 18.4 & 6.05 & 6.0 \\
\hline $4 d$ & $\begin{array}{c}\mathrm{C}_{22} \mathrm{H}_{20} \mathrm{~N}_{8} \mathrm{O}_{7} \mathrm{~S} \\
(540)\end{array}$ & 558 & $\begin{array}{l}191- \\
193\end{array}$ & 63 & 48.89 & 48.8 & 3.73 & 3.7 & 20.73 & 20.7 & 5.93 & 5.9 \\
\hline $4 e$ & $\begin{array}{c}\mathrm{C}_{23} \mathrm{H}_{23} \mathrm{~N}_{7} \mathrm{O}_{6} \mathrm{~S} \\
(525)\end{array}$ & 528 & $\begin{array}{l}195- \\
198\end{array}$ & 67 & 52.56 & 52.5 & 4.41 & 4.4 & 18.66 & 18.6 & 6.10 & 6.0 \\
\hline
\end{tabular}

Table-3 Physical and Analytical Data of the Compounds Synthesized (5a-e) as per reported method ${ }^{21}$

\begin{tabular}{|c|c|c|c|c|c|c|c|c|c|c|c|c|}
\hline \multirow{3}{*}{$\begin{array}{c}\text { Comp. } \\
\text { No. }\end{array}$} & \multirow{3}{*}{$\begin{array}{l}\text { Molecular } \\
\text { Formula }\end{array}$} & \multirow{3}{*}{$\begin{array}{c}\text { LC- } \\
\text { MS } \\
\text { Data }\end{array}$} & \multirow{3}{*}{$\underset{{ }^{\circ} \mathrm{C}}{\text { M.P.* }}$} & \multirow{3}{*}{$\begin{array}{c}\text { Yiel } \\
\text { d } \\
\%\end{array}$} & \multicolumn{8}{|c|}{ Elemental Analysis } \\
\hline & & & & & \multicolumn{2}{|c|}{$\mathrm{C} \%$} & \multicolumn{2}{|c|}{$\mathbf{H \%}$} & \multicolumn{2}{|c|}{$\mathbf{N \%}$} & \multicolumn{2}{|c|}{$\mathbf{S \%}$} \\
\hline & & & & & Calcd. & Found & Calcd. & Found & Calcd. & Found & Calcd. & Found \\
\hline $5 \mathbf{a}$ & $\begin{array}{c}\mathrm{C}_{29} \mathrm{H}_{27} \mathrm{~N}_{7} \mathrm{O}_{6} \mathrm{~S} \\
(601)\end{array}$ & 614 & $\begin{array}{c}196- \\
198\end{array}$ & 60 & 57.90 & 57.8 & 4.49 & 4.4 & 16.30 & 16.2 & 5.32 & 5.3 \\
\hline $5 \mathbf{b}$ & $\begin{array}{c}\mathrm{C}_{30} \mathrm{H}_{29} \mathrm{~N}_{7} \mathrm{O}_{6} \mathrm{~S} \\
(615)\end{array}$ & 627 & $\begin{array}{l}202- \\
203\end{array}$ & 59 & 58.53 & 58.5 & 4.71 & 4.7 & 15.93 & 15.9 & 5.20 & 5.1 \\
\hline $5 c$ & $\begin{array}{c}\mathrm{C}_{29} \mathrm{H}_{26} \mathrm{~N}_{7} \mathrm{O}_{6} \mathrm{SCl} \\
(635)\end{array}$ & 650 & $\begin{array}{l}204- \\
205\end{array}$ & 57 & 54.80 & 54.8 & 4.09 & 4.0 & 15.43 & 15.4 & 5.03 & 5.0 \\
\hline $5 d$ & $\begin{array}{c}\mathrm{C}_{29} \mathrm{H}_{26} \mathrm{~N}_{8} \mathrm{O}_{8} \mathrm{~S} \\
(646)\end{array}$ & 666 & $\begin{array}{c}214- \\
215\end{array}$ & 61 & 53.86 & 53.8 & 4.02 & 4.0 & 17.33 & 17.3 & 4.95 & 4.9 \\
\hline $5 e$ & $\begin{array}{c}\mathrm{C}_{30} \mathrm{H}_{29} \mathrm{~N}_{7} \mathrm{O}_{7} \mathrm{~S} \\
(631)\end{array}$ & 649 & $\begin{array}{l}208- \\
209\end{array}$ & 64 & 57.05 & 57.0 & 4.59 & 4.5 & 15.53 & 15.5 & 5.07 & 5.0 \\
\hline
\end{tabular}

\section{BIOLOGICAL SCREENING}




\section{International Advanced Research Journal in Science, Engineering and Technology}

Vol. 6, Issue 1, January 2019

Antibacterial activities: Antibacterial activities of prepared compounds were studied against gram-positive Bacteria and gram-negative Bacteria at a concentration of $50 \mu \mathrm{g} / \mathrm{ml}$ by agar cup plate method. ${ }^{22}$ Methanol system was used as control in this method. Under similar conditions, using tetracycline as a standard for comparison The percentage area of inhibition of measured. Compounds $\mathbf{5 e}$ and $\mathbf{4 e}$ were found more toxic for microbes. Other compounds found to be less or moderate active than tetracycline. (Table -4$)$

Table-4 Antibacterial Activity of Compounds (4a-e) and (5a-e)

\begin{tabular}{|c|c|c|c|c|c|}
\hline \multirow{3}{*}{$\begin{array}{l}\text { Comp. } \\
\text { No. }\end{array}$} & \multicolumn{5}{|c|}{ Zone of Inhibition(mm) } \\
\hline & \multicolumn{2}{|c|}{ Gram +ve } & \multicolumn{3}{|c|}{ Gram -ve } \\
\hline & $\begin{array}{l}\text { Bacillus } \\
\text { Subtilis }\end{array}$ & $\begin{array}{c}\text { Staphylococcus } \\
\text { aureus }\end{array}$ & $\begin{array}{c}\text { Kllebsiella } \\
\text { promioe }\end{array}$ & $\begin{array}{c}\text { Salmonella } \\
\text { Typhl }\end{array}$ & E.coil \\
\hline $4 \mathbf{4 a}$ & 58 & 43 & 58 & 46 & 58 \\
\hline $4 \mathrm{~b}$ & 54 & 48 & 62 & 58 & 60 \\
\hline $4 c$ & 57 & 47 & 73 & 45 & 59 \\
\hline 4d & 69 & 44 & 78 & 64 & 62 \\
\hline $4 e$ & 70 & 50 & 81 & 72 & 66 \\
\hline $5 \mathbf{a}$ & 60 & 44 & 60 & 47 & 59 \\
\hline $5 \mathbf{b}$ & 55 & 49 & 63 & 59 & 61 \\
\hline $5 \mathbf{c}$ & 59 & 49 & 74 & 46 & 62 \\
\hline 5d & 70 & 47 & 80 & 66 & 63 \\
\hline $5 e$ & 72 & 51 & 82 & 74 & 69 \\
\hline Tetracycline & 79 & 55 & 87 & 76 & 72 \\
\hline
\end{tabular}

Antifungal activity: The fungicidal activity of prepared compounds (5a-e) and (4a-e) was studied at 1000 ppm concentration in vitro plant pathogenic organisms listed in Table-4. The antifungal activities of all the samples were measured on each of these plant pathogenic strains on potato dextrose agar (PDA) medium. Such a PDA medium contained potato $200 \mathrm{gms}$, dextrose $20 \mathrm{gms}$, agar $20 \mathrm{gms}$ and water 1 litre five days old cultures were employed. The compounds to be tested were suspended (1000ppm) in a PDA medium and autoclaved at $120^{\circ} \mathrm{C}$ for $15 \mathrm{~min}$. at 15 atm pressure. These medium were poured into sterile Petiri plate and the organisms were inoculated after cooling the Petri plate. The percentage inhabitation for fungi was calculated after 5 days using the formula given below.

\section{Percentage of inhibition $=100(X-Y) / X$}

Where, X: Area of colony in control plate

Y: Area of colony in test plate

The fungicidal activity all compounds (5a-e) and (4a-e) are shown in Table-5.

Table-5 Antifungal Activity of Compounds (4a-e) and (5a-e)

\begin{tabular}{|c|c|c|c|c|}
\hline \multicolumn{5}{|c|}{ Zone of Inhibition at 1000 ppm (\%) } \\
\hline $\begin{array}{c}\text { Comp. } \\
\text { No. }\end{array}$ & $\begin{array}{c}\text { Botrydepladia } \\
\text { Thiobromine }\end{array}$ & $\begin{array}{c}\text { Nigrosspora } \\
\text { Sp. }\end{array}$ & $\begin{array}{c}\text { Penicillium } \\
\text { Expansum }\end{array}$ & $\begin{array}{c}\text { Rhizopus } \\
\text { Nigricuns }\end{array}$ \\
\hline 4a & 62 & 73 & 74 & 54 \\
\hline 4b & 73 & 67 & 63 & 71 \\
\hline 4c & 56 & 65 & 55 & 72 \\
\hline 4d & 67 & 68 & 68 & 67 \\
\hline 4e & 74 & 80 & 74 & 76 \\
\hline 5a & 63 & 75 & 76 & 55 \\
\hline 5b & 75 & 69 & 66 & 73 \\
\hline 5c & 58 & 66 & 57 & 74 \\
\hline 5d & 69 & 69 & 69 & 69 \\
\hline 5e & 75 & 82 & 76 & 78 \\
\hline
\end{tabular}

IV.

RESULTS AND DISCUSSIONS

The ethyl-3-oxo-2-(2-(4-sulfamoylphenyl)hydrazono)butanoate (2) react with 6-methyl-2-oxo-4-subsitutedphenyl1,2,3,4-tetrahydro pyrimidine-5-carbohydrazide (3a-e) to gane 4-(2-(3-methyl-1-(6-methyl-2-oxo-4-subsitutedphenyl1,2,3,4-tetrahydro pyrimidine -5-carbonyl)-5-oxo-1H-pyrazol-4 $(5 \mathrm{H})$-ylidene) hydrazinyl) benzene sulfonamide (4a-e), which gives 4-(2-(1-(4-([1,1'-biphenyl]-4-yl)-3-(hydroxy(phenyl)aryl)-6-methyl-2-oxo-1,2,3,4-tetrahydropyrimidine-5carbonyl)-3-methyl-5-oxo-1H-pyrazol-4(5H)-ylidene) hydrazinyl) benzenesulfonamide (5a-e) on reaction with benzaldehyde. 


\title{
International Advanced Research Journal in Science, Engineering and Technology
}

\author{
Vol. 6, Issue 1, January 2019
}

The structures of (3a-e) were confirmed by elemental analysis and IR spectra showing an absorption bands at $3500(\mathrm{~N}-\mathrm{H}), 3030-3080 \mathrm{~cm}^{-1}(\mathrm{C}-\mathrm{H}$ of $\mathrm{Ar}), 1680 \mathrm{~cm}^{-1}$ (CONH), 2950, $1370 \mathrm{~cm}^{-1}\left(-\mathrm{CH}_{3}, \mathrm{CH}_{2}\right), 1080(-\mathrm{Cl}), 1555,1375(-$ $\left.\mathrm{NO}_{2}\right), 1695-1750 \mathrm{~cm}^{-1}(\mathrm{C}=\mathrm{O}) .{ }^{1} \mathrm{H}$ NMR $\left(400 \mathrm{MHz}, \mathrm{DMSO}-\mathrm{d}_{6}, \delta / \mathrm{ppm}\right): 11.8-11.9(\mathrm{~s}, 3 \mathrm{H}, \mathrm{NH}), 2.52(\mathrm{t}, 3 \mathrm{H}, \mathrm{CH} 3)$, 5.42(s,1H,CH) (3a): 7.23-7.37 (s,5H, ArH); (3b): $1.26 \quad\left(\mathrm{~s}, 3 \mathrm{H}, \mathrm{CH}_{3}\right) \quad$ ， 7.20-7.29 (s,4H,ArH) ; (3c): 7.197.22(s,4H,ArH);(3d):7.18-7.24(s,4H,ArH); $(3 e): 4.21\left(\mathrm{~s}, 3 \mathrm{H}, \mathrm{CH}_{3}\right), 7.22-7.26(\mathrm{~s}, 4 \mathrm{H}, \mathrm{ArH}) . \mathrm{The} \mathrm{C}, \mathrm{H}, \mathrm{N}$ analysis data of all compounds are presented in Table-1.

The IR spectra of (4a-e) are 1620-1630 cm $\mathrm{cm}^{-1}(\mathrm{C}=\mathrm{N}), 3030-3080 \mathrm{~cm}^{-1}\left(\mathrm{C}-\mathrm{H}\right.$ of Ar.), 2960, $1370 \mathrm{~cm}^{-1}\left(-\mathrm{CH}_{3}\right), 1710-$ $1760(\mathrm{C}=\mathrm{O}), 1380,1160\left(\mathrm{SO}_{2}\right), 1080(-\mathrm{Cl}), 1555,1375\left(-\mathrm{NO}_{2}\right), 3330$ and $3155 \mathrm{~cm}^{-1}(\mathrm{NH})$ and 1585,1548, and $1530 \mathrm{~cm}^{-1}$ $(\mathrm{C}=\mathrm{C}) .{ }^{1} \mathrm{H}$ NMR $\left(400 \mathrm{MHz}, \mathrm{DMSO}-\mathrm{d}_{6}, \delta / \mathrm{ppm}\right): 11.8-11.9,8.4(\mathrm{~s}, 3 \mathrm{H}, \mathrm{NH}), 7.58\left(\mathrm{~s}, 2 \mathrm{H}, \mathrm{NH}_{2}\right), 2.52-240\left(\mathrm{~s}, 6 \mathrm{H}, \mathrm{CH}_{3}\right)$, 5.42(s,1H, CH), 7.01-7.20(m,4H,Ar-H), (4a): 7.23-7.37 (s,5H, ArH); (4b): $1.26\left(\mathrm{~s}, 3 \mathrm{H}, \mathrm{CH}_{3}\right), 7.20-7.29$ (s,4H,ArH) ; (4c): 7.19-7.22(s,4H,ArH); (4d): 7.18-7.24(s,4H,ArH); (4e): 4.21(s, 3H, CH $\left.\mathrm{CH}_{3}\right)$ 7.22-7.26(s,4H,ArH).The C, H, N analysis data of all compounds are presented in Table -2 .

The IR spectra of (5a-e) are 1620-1630 $\mathrm{cm}^{-1}(\mathrm{C}=\mathrm{N}), 3420 \mathrm{~cm}^{-1}(\mathrm{OH}), 3030-3080 \mathrm{~cm}^{-1}$ (C-H of Ar.), $2960,1370 \mathrm{~cm}^{-1}(-$ $\left.\mathrm{CH}_{3}\right), 1710-1760(\mathrm{C}=\mathrm{O}), 1380,1160\left(\mathrm{SO}_{2}\right), 1080(-\mathrm{Cl}), 1555,1375\left(-\mathrm{NO}_{2}\right), 3330$ and $3155 \mathrm{~cm}^{-1}(\mathrm{NH})$ and 1585,1548 , and $1530 \mathrm{~cm}^{-1}(\mathrm{C}=\mathrm{C}) .{ }^{1} \mathrm{H}$ NMR $\left(400 \mathrm{MHz}, \mathrm{DMSO}-\mathrm{d}_{6}, \delta / p p m\right): 11.8-11.9,8.4(\mathrm{~s}, 2 \mathrm{H}, \mathrm{NH}), 7.58\left(\mathrm{~s}, 2 \mathrm{H}, \mathrm{NH}_{2}\right), 2.52-240(\mathrm{~s}, 6 \mathrm{H}$, $\left.\mathrm{CH}_{3}\right), 5.42(\mathrm{~s}, 1 \mathrm{H}, \mathrm{CH}), 7.01-7.20(\mathrm{~m}, 9 \mathrm{H}, \mathrm{Ar}-\mathrm{H}), 6.67(\mathrm{~s}, 1 \mathrm{H}, \mathrm{CH}), 3.72(\mathrm{~s}, 1 \mathrm{H}, \mathrm{OH}),(\mathbf{5 a}): 7.23-7.37$ (s, 5H, ArH); (5b): 1.26 $\left(\mathrm{s}, 3 \mathrm{H}, \mathrm{CH}_{3}\right), 7.20-7.29$ (s,4H,ArH) ; (5c): 7.19-7.22(s,4H,ArH); (5d): 7.18-7.24(s,4H,ArH); (5e): 4.21(s, 3H, $\left.\mathrm{CH}_{3}\right)$, 7.227.26(s,4H,ArH).The $\mathrm{C}, \mathrm{H}, \mathrm{N}$ analysis data of all compounds are presented in Table -3 .

The assessment of data predicts that the elemental contents are consistence with the predicted structure shown in Scheme-1.The IR data also direct for assignment of the predicted structure. The LC-MS of compounds shows the peak of $\mathrm{M}^{+}$ion which is consistent of molecular weight of respect sample. All these facts confirm the structures 5a-e \& $4 \mathbf{a}-\mathbf{e}$.

\section{CONCLUSION}

The examination of antibacterial activity data reveals that the compounds $\mathbf{4 e}$ and $\mathbf{5 e}$ found more active against the grampositive and gram-negative bacteria.

\section{REFERENCES}

[1]. J. Feng, S. Zhang, W. Shi and Y. Zhang, Antibiotics,6(1),10 (2017).

[2]. J.Shaah, B.Patel and S. Patel, Journal of Saudi Chemical Society, 17,307(2013).

[3]. M. S. Mohamed, S. M. Awad, Y.M.Zohny and Z. M. Mohamed, Pharmacophore, 3(1), 6217(2012).

[4]. H.Alsharifi and H.S. Patel, International Journal of Science Innovations and Discoveries,

[5]. 2(4), 408(2012).

[6]. P.A. Patil, R. P. Bhole, R. V. Chikhal and K. P. Bhusari, Int.J.Chem Tech Res., 1(2),373 (2009).

[7]. G.Malhotra and Y.K. Srivastava, J.Chem.Bio.Phy.Sci., 1(1),45, (2011).

[8]. J.Shaah, B.Patel and S. Patel, Bulgarian Chemical Communications, 42(4), 474,(2010).

[9]. I. M. El-Deeb, S. H. Lee, Bioorg. Med. Chem., 18,3860, (2010).

[10]. T.Yang, H.He, W.Ang, Y.Yang, J.Yang, Y.Lin, H.Yang, W.Pi, Z.Li, Y.Zhao,Y. Luo and

[11]. Y.Wei,Molecules, 17,2351, (2012).

[12]. C. Li, J.K. Dutra, B. Rast, R.M. Crosson, K.M. Callaghan, S.B. Seibel and A. Silvia,

[13]. Bioorg Med Chem. Lett.,14, 95,(2004).

[14]. A.A. Bekhit and H.T. Fahmy, Arch Pharm (Weinheim)., 336,111, (2003).

[15]. S.B. Kativar,, K. Srivastava,, S.K. Puri and P.M. Chauhan, Bioorg Med Chem Lett.,

[16]. 15,4957(2015).

[17]. S. Kuettel, A. Zambon, M. Kaiser, R. Brun, L. Scapozza and R. Perozzo,, J Med. Chem.,

[18]. 50,5833(2017).

[19]. S. J.Shah, Heterocyclic Letters, 7(2),405,(2017).

[20]. S. Gemma, G. Kukreja, M. Persico, M. Romano, M. Altarelli, L. Savini, G. Campiani, E. Fattorusso and N. Basilico, Bioorg. Med. Chem. Lett.,16, 5384,(2006).

[21]. M. R. Shiradkar, K. K. Murahari, C. C. Kalyan, D. Panchal, R. Kaur, P. Burange,

[22]. J. Ghogare, V. Mokale, M. Raut, Bioorg. Med. Chem.,15,3997,(2017).

[23]. M. R. Rao, K. Hart, N. Devanna and K. B. Chandrasekhar, Asian J.Chem.,20,1402, (2018).

[24]. P. Patel, "Synthesis, Characterization and Antimicrobial Screening of Novel Arylazopyrazoles dissertation,Sardar Patel University,V.V.Nagar,43,(2013).

[25]. R. Tayebee and M. Ghadamghai, Arabian J. of Chemistry, 10(1),5757-5764(2017).

[26]. Sanakausar R. Shaikh and G. M. Nazeruddin, J. Chem. Phara. Res., 6(12),505-534, (2014).

[27]. 21.Nafisa Anjum, Bangladesh Uni.of Eng. \& Tech. Dhaka-1000. Development of facile synthetic routes for the synthesis of dihydropyrimidone derivatives, 23 march 2017,lib. Buet. Ac. Bd

[28]. A.L. Barry,The Antimicrobial Susceptibility Test:Principal and Practices,4th ed., edited by Illuslea and Feger,Philadelphia,180(1976). 